\title{
Strain heterogeneity, cooccurrence network, taxonomic composition and functional profile of the healthy ocular surface microbiome
}

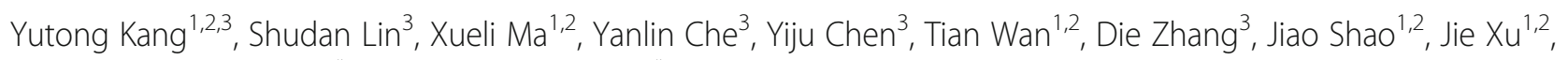
Yi Xu ${ }^{1,2}$, Yongliang Lou ${ }^{3 *}$ and Meiqin Zheng ${ }^{1,2,3^{*}}$ (D)

\begin{abstract}
Background: There is growing evidence indicating that the microbial communities that dwell on the human ocular surface are crucially important for ocular surface health and disease. Little is known about interspecies interactions, functional profiles, and strain heterogeneity across individuals in healthy ocular surface microbiomes.

Methods: To comprehensively characterize the strain heterogeneity, cooccurrence network, taxonomic composition and functional profile of the healthy ocular surface microbiome, we performed shotgun metagenomics sequencing on ocular surface mucosal membrane swabs of 17 healthy volunteers.

Results: The healthy ocular surface microbiome was classified into 12 phyla, 70 genera, and 140 species. The number of species in each healthy ocular surface microbiome ranged from 6 to 47, indicating differences in microbial diversity among individuals. The species with high relative abundances and high positivity rates were Streptococcus pyogenes, Staphylococcus epidermidis, Propionibacterium acnes, Corynebacterium accolens, and Enhydrobacter aerosaccus. A correlation network analysis revealed a competitive interaction of Staphylococcus epidermidis with Streptococcus pyogenes in ocular surface microbial ecosystems. Staphylococcus epidermidis and Streptococcus pyogenes revealed phylogenetic diversity among different individuals. At the functional level, the pathways related to transcription were the most abundant. We also found that there were abundant lipid and amino acid metabolism pathways in the healthy ocular surface microbiome.
\end{abstract}

Conclusion: This study explored the strain heterogeneity, cooccurrence network, taxonomic composition, and functional profile of the healthy ocular surface microbiome. These findings have important significance for the future development of probiotic-based eye therapeutic drugs.

Keywords: Healthy ocular surface microbiome, Cooccurrence network, Functional composition, Strain level

\footnotetext{
* Correspondence: louyongliang2013@163.com; zmq@eye.ac.cn

${ }^{3}$ Wenzhou Key Laboratory of Sanitary Microbiology, Key Laboratory of

Laboratory Medicine, Ministry of Education, School of Laboratory Medicine

and Life Sciences, Wenzhou Medical University, Wenzhou, Zhejiang 325035,

China

${ }^{1}$ Eye Hospital and School of Ophthalmology and Optometry, Wenzhou

Medical University, Wenzhou, Zhejiang, China

Full list of author information is available at the end of the article
}

\section{$\triangle B M C$}

(c) The Author(s). 2021 Open Access This article is licensed under a Creative Commons Attribution 4.0 International License, which permits use, sharing, adaptation, distribution and reproduction in any medium or format, as long as you give appropriate credit to the original author(s) and the source, provide a link to the Creative Commons licence, and indicate if changes were made. The images or other third party material in this article are included in the article's Creative Commons licence, unless indicated otherwise in a credit line to the material. If material is not included in the article's Creative Commons licence and your intended use is not permitted by statutory regulation or exceeds the permitted use, you will need to obtain permission directly from the copyright holder. To view a copy of this licence, visit http://creativecommons.org/licenses/by/4.0/ The Creative Commons Public Domain Dedication waiver (http://creativecommons.org/publicdomain/zero/1.0/) applies to the data made available in this article, unless otherwise stated in a credit line to the data. 


\section{Background}

Human mucosal surfaces are normally colonized by diverse microbial flora [1]. As the most exposed mucosal tissue of the human body, the ocular surface is susceptible to insult by environmental factors, such as microorganisms [2]. Although a healthy ocular surface can live in harmony with symbiotic microorganisms, microbial community imbalance or transient flora increases on the ocular surface may lead to diseases [3]. Bacteria are considered major contributors to ocular infections worldwide. Ocular infections, if not promptly treated, can cause vision impairment and blindness [4]. In the $\mathrm{Hu}-$ man Microbiome Project, much effort has been applied to characterize the human mucosal microbiome, spanning the gut, mouth, respiratory tract, skin, and urogenital tract [5]. However, in the field of visual research, this aspect is still in its infancy.

Previous culture-based surveys indicated that the microbial flora colonizing the ocular surface is dominated by gram-positive Firmicutes [6]. Whereas conventional methods have difficulty detecting microorganisms that are rarely encountered, grow slowly, and cannot be cultured, next-generation sequencing (NGS) technologies have provided a much more detailed picture of the healthy ocular surface microbiome [7-11]. Although the precise distribution of each phylum is different between individuals, the most consistent contributors on the ocular surface are Proteobacteria, Firmicutes, and Actinobacteria $[3,7,10,12,13]$.

A number of studies in the intestine and other microbiome sites have shown that cohabiting microorganisms within a microbiome maintain a stable state of competition and cooperation, such as competing with one another for or mutually utilizing resources, nutrition, and space $[14,15]$. The abundance of each member of the microbiome is constrained by the optimal ratio to maintain homeostasis [14]. Interactions among microbial species are crucial for the sustainability of various ecosystems [16]. However, our current understanding of many interaction relationships among the components of the ocular surface microecosystem is still very limited.

The microbial communities that dwell on the human ocular surface are important for ocular surface health and disease [17-22]. The microbial flora populating the human ocular surface has been characterized with NGS, but few studies have focused on strain-level resolution. Although high-quality studies have been conducted on the genetic variation and population structure of humans and how population heritability is shaped, the variation and structure of bacterial cells residing in the human body are relatively unknown [23-25]. Strainlevel analysis of the gut microbiome has shown that in common species, different strains of species are associated with different individuals [26].
Most previous studies used mainly 16S rRNA gene sequencing, which did not allow for confident classification of the microbiota to the level of (sub)species [27], and 16S rRNA gene sequencing cannot provide data regarding functional genes and can indicate only the potential functionality of microbial communities. Our metagenome sequencing data provide more direct functional information and higher taxonomic resolution for a comprehensive understanding of the taxonomic and functional compilations of the healthy ocular surface microbiome. Species interactions and strain heterogeneity among individuals have received little attention in past studies based on shotgun metagenomics sequencing for the characterization of normal ocular surface microecology $[28,29]$. Here, we demonstrate the individual differences in Staphylococcus epidermidis and Streptococcus pyogenes at the strain level. We also found the existence of a competing relationship between Staphylococcus epidermidis and Streptococcus pyogenes in the cooccurrence network.

\section{Methods \\ Ethics approval and consent to participate}

This study was approved by the Ethics Committee of the Eye Hospital of Wenzhou Medical University (number: KYK [2017] 23) and adhered to the tenets of the Declaration of Helsinki. All subjects provided written informed consent at the time of sample collection.

\section{Sample collection and processing}

A total of 8 male subjects (age $41.6 \pm 13.7$ years) and 9 female subjects (age $43 \pm 13.3$ years) with healthy ocular surfaces were recruited from communities across Zhejiang, China. All subjects received systematic eye examinations by the same ophthalmologist before sample collection. In addition, all subjects were requested to fill in an ocular surface disease index (OSDI) screening questionnaire to evaluate ocular discomfort. The overall OSDI screening questionnaire scores defined the ocular surface as normal (0-12 points) [30]. The overall OSDI screening questionnaire scores of all subjects in this study were 5 or less. The exclusion criteria of the study were as follows: (i) history of systemic or (ii) ocular diseases or (iii) contact lens wearing and (iv) topical or systemic antibiotics, steroid, any eye drop (prescribed or over the counter) or probiotic treatment within 6 months. The subject's eye was administered sterile topical proparacaine. After topical anesthesia for 1 to $3 \mathrm{~min}$, the subject looked upward for sample collection. Samples were taken from the ocular surface mucosal tissues (upper and lower palpebral, caruncle, and conjunctival fornix) using flocked swabs and stored in a Copan ESwab transport system (Copan Diagnostics Inc., Murrieta, CA) on ice blocks. Upon return to the laboratory, 
the swabs were frozen at $-80^{\circ} \mathrm{C}$ until further processing. Genomic DNA was extracted from the swabs using pathogen lysis tubes L (QIAGEN, Hilden, Germany) and a QIAamp UCP Pathogen Mini Kit (QIAGEN, Hilden, Germany) according to the manufacturer's instructions. The DNA concentration was measured using a Qubit $^{\circ}$ 2.0 Fluorometer.

\section{Prevention of contamination and negative controls}

To avoid contamination during sample collection, sample collections were carried out in an ophthalmic treatment room sterilized by ultraviolet light. Unused clean swabs were exposed to the sampling environment and waved in air for $10 \mathrm{~s}$ to collect field controls. Extraction controls were generated during DNA extraction to monitor for reagent contamination. Unused sterile flocked swabs moistened with sterile topical proparacaine were processed as anesthetic controls for DNA extraction. No DNA was detected in the field controls, extraction controls, or anesthetic controls using a Qubit $^{\circ}$ dsDNA Assay Kit and a Qubit ${ }^{\circ}$ 2.0 Fluorometer.

We amplified the variable 4 (V4) region of the $16 \mathrm{~S}$ ribosomal RNA gene extracted from the field controls, extraction controls, anesthetic controls, and actual samples using the universal primers $515 \mathrm{~F}$ and $806 \mathrm{R}$. PCR reagents without template DNA were used for PCR amplification as a negative control. The amplification system was $20 \mu \mathrm{l}$, consisting of $4 \mu \mathrm{l} 5^{*}$ FastPfu buffer, $2 \mu \mathrm{l}$ $2.5 \mathrm{mM}$ dNTPs, $0.8 \mu \mathrm{l}$ primers $(5 \mu \mathrm{M}), 0.4 \mu \mathrm{l}$ FastPfu polymerase, and 10 ng DNA template. Reaction mixtures were incubated for predenaturation at $95^{\circ} \mathrm{C}$ for $3 \mathrm{~min}$, 27 thermal cycles (denaturation at $95^{\circ} \mathrm{C}$ for $30 \mathrm{~s}$, annealing at $55^{\circ} \mathrm{C}$ for $30 \mathrm{~s}$, and extension at $72^{\circ} \mathrm{C}$ for $30 \mathrm{~s}$ ), and extension at $72{ }^{\circ} \mathrm{C}$ for $10 \mathrm{~min}$ (PCR instrument: $\mathrm{ABI}$ GeneAmp 9700). Amplification products of the $16 \mathrm{~S}$ rRNA gene V4 DNA region were visualized by agarose gel electrophoresis. Bright bands were observed at $\sim 290$ bp in actual samples; no band was found in field controls, extraction controls, anesthetic controls, or PCR negative controls. Sequencing was performed on field controls, extraction controls, anesthetic controls, and PCR negative controls and did not yield any reads.

\section{Shotgun metagenomics sequencing}

The above samples were used for shotgun metagenomics sequencing. Paired-end sequencing $(150 \mathrm{bp} \times 2)$ was performed on the HiSeq X10 platform (Novogene Co., Ltd., Beijing, China). The quality of raw reads was assessed using FastQC software according to a previously described in-house bioinformatics pipeline [31]. After the adapter sequences were trimmed by the Cutadapt tool (http://code.google.com/p/cutadapt/), low-quality reads were removed using Trim Galore [32], and the remaining high-quality reads were visualized using
SplicingViewer [33]. Bowtie2 was used to map trimmed reads to the human reference genome (hg19) to generate BAM files [34]. Aligned reads were removed using SAMtools to obtain clean nonhuman sequences [35]. MetaPhlAn2 was executed to generate taxonomic profiles, with default parameters [36]. Strain-level profiling was performed with StrainPhlAn [37]. The remaining metagenomic sequences for each sample were assembled with Megahit [38]. The contigs were submitted to Prokka for gene prediction [39]. Quantification of predicted genes was performed with Salmon [40]. Subsequently, redundant amino acid sequences were removed by using CD-HIT with a sequence identity threshold of 90\% [41]. Functional annotations and transcription factor prediction were implemented using eggNOGmapper [42], CollecTF [43], and ArchaeaTF [44]. Figures were visualized with $R$ (version 3.6.2). The $R$ vegan package was used to calculate the Shannon diversity index, inverse Simpson index, Bray-Curtis dissimilarity, and Jaccard index at the species level. Pairwise comparisons of alpha diversity indices were performed using the Wilcoxon rank-sum test. Permutational multivariate analysis of variance (PERMANOVA) of Bray-Curtis distances and Jaccard distances were performed for statistical analysis of beta diversity. Principal coordinate analysis $(\mathrm{PCoA})$ was used to visualize the resulting distance matrix.

\section{Results}

\section{Taxonomic composition}

Illumina sequencing of all samples produced 1.35 billion reads. After filtering out reads matching the human genome sequence, an average of approximately 2.18 million microbial reads from each sample were obtained for further analysis. The healthy ocular surface microbiome was classified into 12 phyla, 20 classes, 29 orders, 50 families, 70 genera, and 140 species among all subjects (see Supplemental Figure 1). At the phylum level, 7 phyla had an average relative abundance $>1 \%$, namely, Actinobacteria, Bacteroidetes, Deinococcus-Thermus, Firmicutes, Proteobacteria, Eukaryota-noname, and Virusesnoname (see Supplemental Figure 2a). The top three phyla (Firmicutes (average: 45.02\%), Actinobacteria (28.45\%), and Proteobacteria (16.17\%) accounted for the majority. Interestingly, the healthy ocular surface microbiome in some samples was dominated by a single phylum. For instance, in the ocular microbiota of CON3 and CON10, Firmicutes accounted for 80.76 and $83.89 \%$, respectively.

At the genus level, the 15 genera with more than 1\% average relative abundance were Streptococcus, Corynebacterium, Propionibacterium, Staphylococcus, Neisseria, Morganella, Escherichia, Shigella, Siphoviridae, Acinetobacter, Finegoldia, Anelloviridae, Alphatorquevirus, 
Brevundimonas, and Enhydrobacter (see Supplemental Figure 2b). Streptococcus (average: 24.62\%), Staphylococcus (14.15\%), Propionibacterium (12.93\%), and Corynebacterium (9.05\%) were the top four genera. Similarly, the subjects CON10 and CON3 showed extreme dominance by Streptococcus, accounting for 83.89 and $79.49 \%$, respectively.

At the species level, $19.59 \pm 11.34$ (range, 6-47) species were detected in the healthy ocular surface microbiome samples from each subject. No species was detected simultaneously in any subject (see Supplemental Figure 3). The taxonomic composition was unique to each sample. Among all species, Propionibacterium acnes, Staphylococcus epidermidis, Enhydrobacter aerosaccus, Corynebacterium accolens, Corynebacterium pseudogenitalium, Corynebacterium tuberculostearicum, Streptococcus pyogenes, Anaerococcus prevotii, and Finegoldia magna were detected in $>50 \%$ of healthy volunteers (Fig. 1a). Figure $1 \mathrm{~b}$ shows the distribution and relative abundance of the top 25 species. The five most abundant species were Streptococcus pyogenes, Staphylococcus epidermidis, Propionibacterium acnes, Corynebacterium accolens, and Enhydrobacter aerosaccus.

Bacteria were shared by all subjects, yet fungi and viruses were not found in all healthy ocular surface microbiomes. The positive rate for fungi was $35 \%$, and that for viruses was $41 \%$. The average relative abundances of bacteria, fungi and viruses were 93,2 , and $5 \%$, respectively. The fungal microbiome was classified into 2 phyla (Ascomycota and Basidiomycota) and 4 genera (Yarrowia, Chaetomiaceae, Fusarium, and Malassezia) (Fig. 2a). Seven viruses, namely, Propionibacterium phage PAD20,

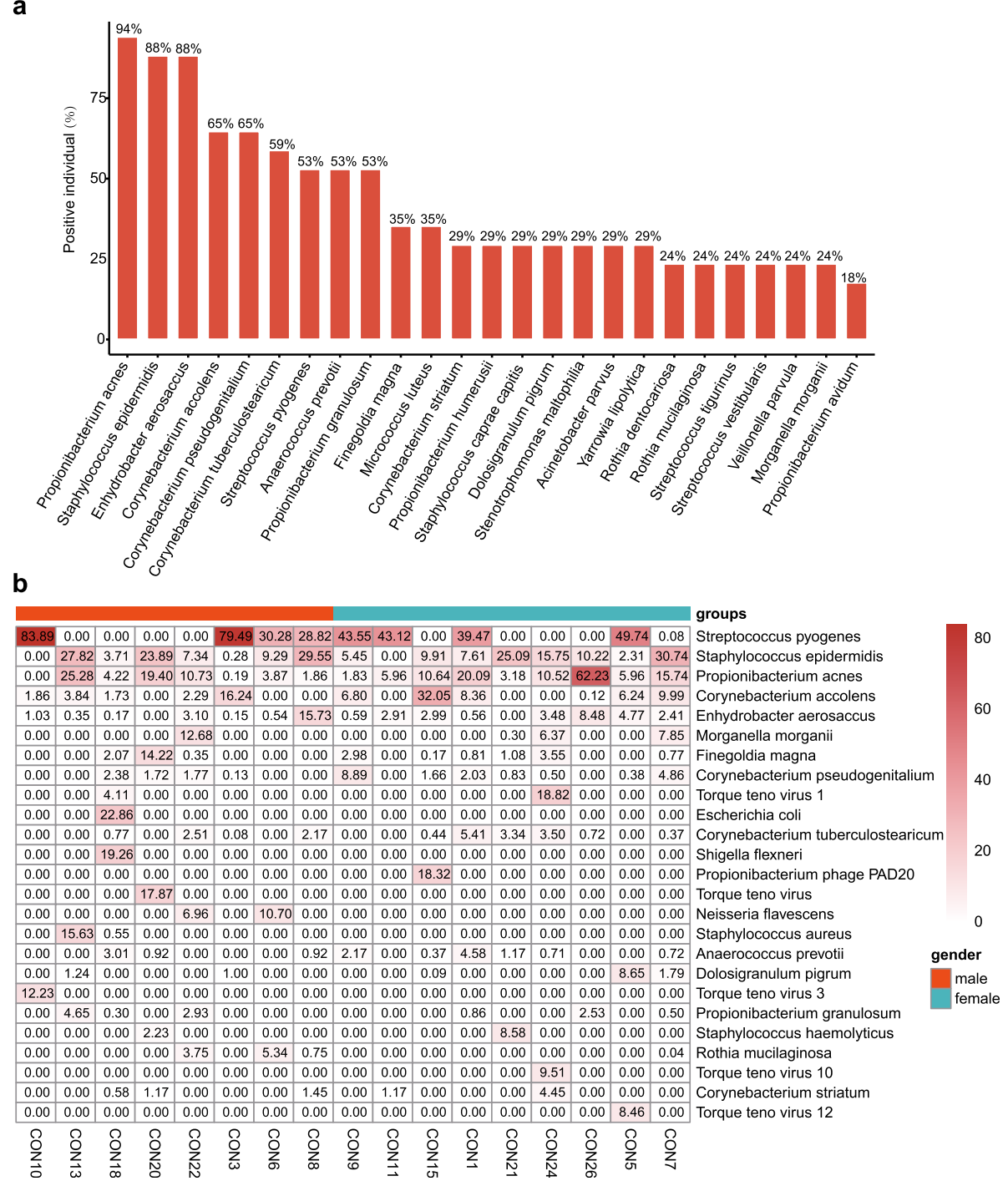

Fig. 1 The positive rate and relative abundance of microbial species in the healthy ocular surface. a Species with a positive rate greater than $10 \%$ are shown. $\mathbf{b}$ The heat map shows the relative abundance of the top 25 species in each sample 
a

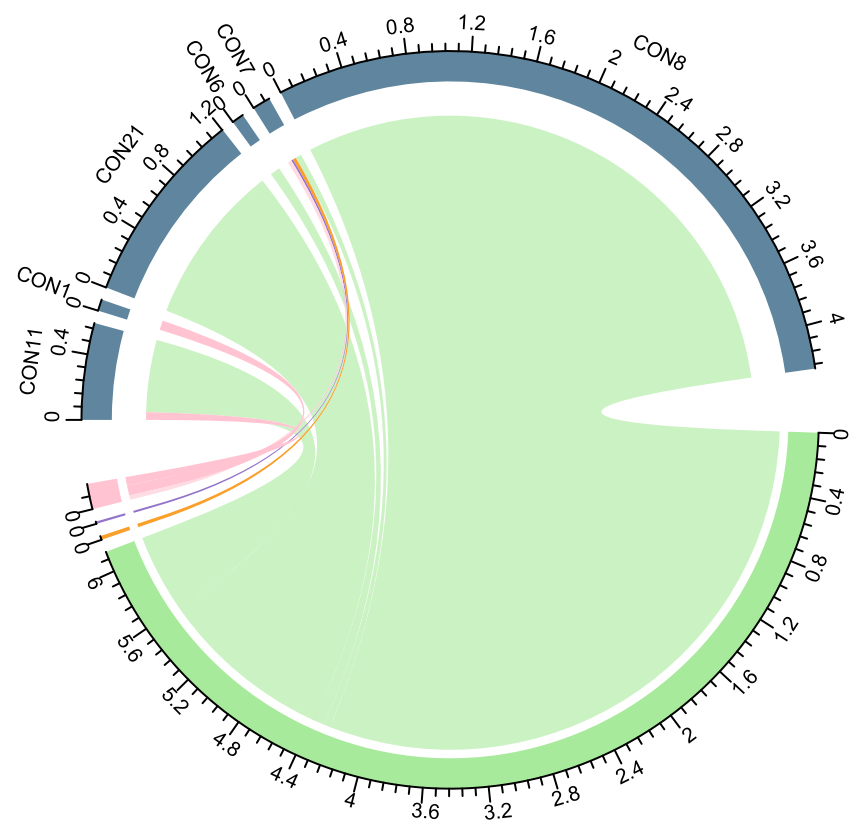

k_Eukaryota.p_Ascomycota.c_Saccharomycetes.o_Saccharomycetales.f_Dipodascaceae.g_Yarrowia.s_Yarrowia lipolytica - k_Eukaryota.p_Ascomycota.c__Sordariomycetes.o_Hypocreales.f_Nectriaceae.g_Fusarium.s Fusarium unclassified

k_Eukaryota.p_Ascomycota.c Sordariomycetes.o Sordariales.f_Chaetomiaceae.g_Chaetomiaceae unclassified
k_Eukaryota.p_Basidiomycota.c_Exobasidiomycetes.o_Malasseziales.f_Malasseziaceae.g_Malassezia.s_Malassezia globosa

b

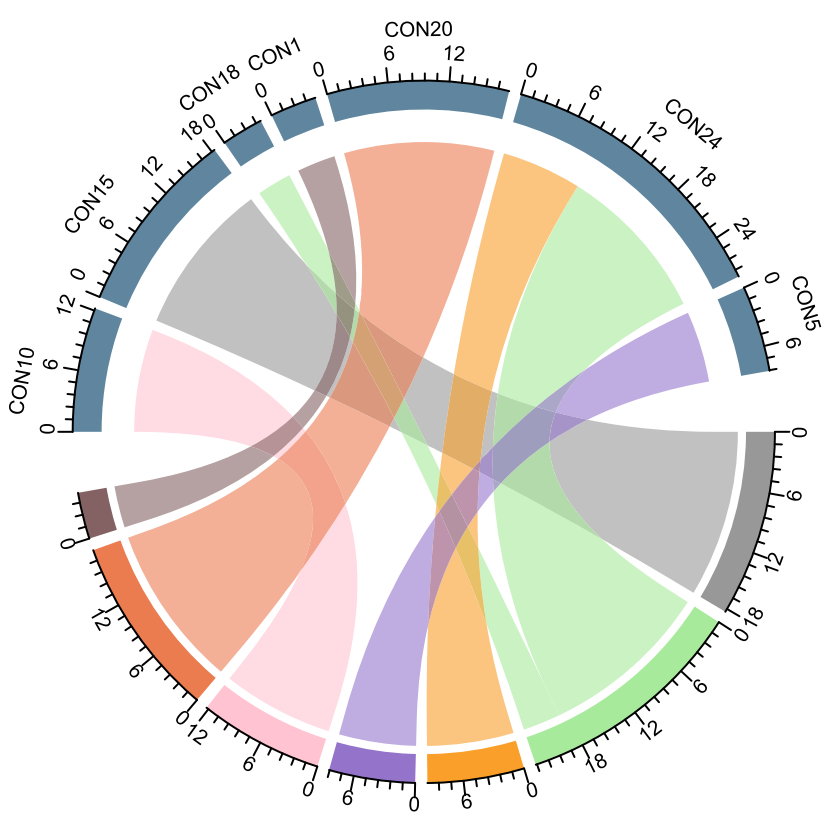

- k_Viruses.p_Viruses_noname.c_Viruses_noname.o_Caudovirales.f_Siphoviridae.g_Siphoviridae_noname.s_Propionibacterium phage PAD20

k_Viruses.p_Viruses_noname.c_Viruses_noname.o_Viruses_noname.f_Anelloviridae.g_Alphatorquevirus.s_Torque teno virus 1

$\mathrm{k}$ _Viruses.p_ Viruses noname.c Viruses noname.o Viruses noname.f Anelloviridae.g Alphatorquevirus.s Torque teno virus 10

- k_Viruses.p_Viruses_noname.c_Viruses_noname.o__Viruses_noname.f_Anelloviridae.g_Alphatorquevirus.s_Torque teno virus 12

k_Viruses.p Viruses noname.c Viruses noname.o Viruses noname.f Anelloviridae.g Alphatorquevirus.s - Torque teno virus 3

- k_Viruses.p_Viruses_noname.c_Viruses_noname.o_Viruses_noname.f_Anelloviridae.g_Anelloviridae_noname.s_Torque teno virus

- k_Viruses.p_Viruses_noname.c_Viruses_noname.o_Viruses_noname.f_Retroviridae.g_Gammaretrovirus.s_Porcine type C oncovirus

Fig. 2 Chord diagram showing the distribution and relative abundance of (a) fungi and (b) viruses in the subjects 
Torque teno virus 1, Torque teno virus 10, Torque teno virus 12, Torque teno virus 3, Torque teno virus, and Porcine type $C$ oncovirus, were detected (Fig. 2b).

\section{Strain heterogeneity}

StrainPhlAn allowed us to study the strain-level features of the healthy ocular surface microbiome, which targets single-nucleotide polymorphisms (SNPs) within cladespecific markers of strains in metagenomes. Among all species, Streptococcus pyogenes and Staphylococcus epidermidis with sufficient coverage were profiled by using the StrainPhlAn method. We constructed a phylogenetic tree of Streptococcus pyogenes (Fig. 3a) covering eight individuals and Staphylococcus epidermidis (Fig. 3b) covering 10 individuals from the metagenomic sequence data. Using SNP-based analysis, considerable strain-level heterogeneity was observed with respect to reference genomes (Streptococcus pyogenes M1 GAS and Staphylococcus epidermidis ATCC 12228). The common species from different individuals formed almost distinct branches, which could indicate the presence of different strains.

For Streptococcus pyogenes and Staphylococcus epidermidis, the strains were separated into two clusters, but neither of the clusters was age- or sex-specific. Staphylococcus epidermidis residing within subject CON7 was closely related to Staphylococcus epidermidis ATCC 12228. Staphylococcus epidermidis strains residing within CON21 and CON22 formed a monophyletic clade. Streptococcus pyogenes M1 GAS clustered together with Streptococcus pyogenes strains residing within CON3 and CON10, which indicated an intimate intraspecies relationship among them. In addition, Streptococcus pyogenes residing within CON3 (Streptococcus pyogenes CON3) branched near the base of Streptococcus pyogenes M1 GAS and Streptococcus pyogenes residing within CON10 (Streptococcus pyogenes CON10). We inferred that Streptococcus pyogenes CON3 likely arose before Streptococcus pyogenes M1 GAS and Streptococcus pyogenes CON10.

\section{Interaction network}

An interaction network was constructed for species with a mean relative abundance greater than $0.1 \%$ that appeared in more than two subjects (Fig. 4a). Streptococcus_mitis_oralis_pneumoniae had the most interactions, suggesting key roles in the network. Streptococcus_mitis_ oralis_pneumoniae exhibited only positive interactions with 7 species, Staphylococcus_caprae_capitis, Rothia mucilaginosa, Haemophilus parainfluenzae, Neisseria meningitidis, Veillonella parvula, Streptococcus vestibularis, and Streptococcus tigurinus. Interestingly, only Staphylococcus epidermidis was negatively related to Streptococcus pyogenes, implying that there may be competitive inhibition between them. Based on this result, subjects were clustered by the k-means algorithm with $\mathrm{k}=2$, using the relative abundance of Streptococcus pyogenes and Staphylococcus epidermidis. Unsupervised clustering generated two distinct clusters: cluster 1 and cluster 2 (Fig. 4b). In cluster 1 , the relative abundance of Streptococcus pyogenes in each sample was $>30 \%$.

\section{The effects of age and sex on the composition of healthy ocular surface microbiomes}

The subjects were grouped by sex (female versus male) and age ( $\leq 40$ versus $>40$ years old) to investigate the influence of age and sex on the normal ocular surface microbiome. Alpha diversity analysis based on the Shannon and inverse Simpson diversity indices revealed that there was no significant variation between sexes (see Supplemental Figure 4a, b) and ages (see Supplemental Figure 5a, b). PCoA based on the BrayCurtis dissimilarity Jaccard index showed no effect on bacterial community structure for sex (see Supplemental Figure 4c, d) or age (see Supplemental Figure $5 c, d)$.

\section{Functional pathways}

At present, the functional compositions of the healthy ocular surface microbiome are still poorly understood. Clusters of Orthologous Groups (COG) analysis revealed


Fig. 3 Maximum-likelihood trees derived from strain variation in individual bacteria inferred using StrainPhIAn. a Streptococcus pyogenes; $\mathbf{b}$ Staphylococcus epidermidis 


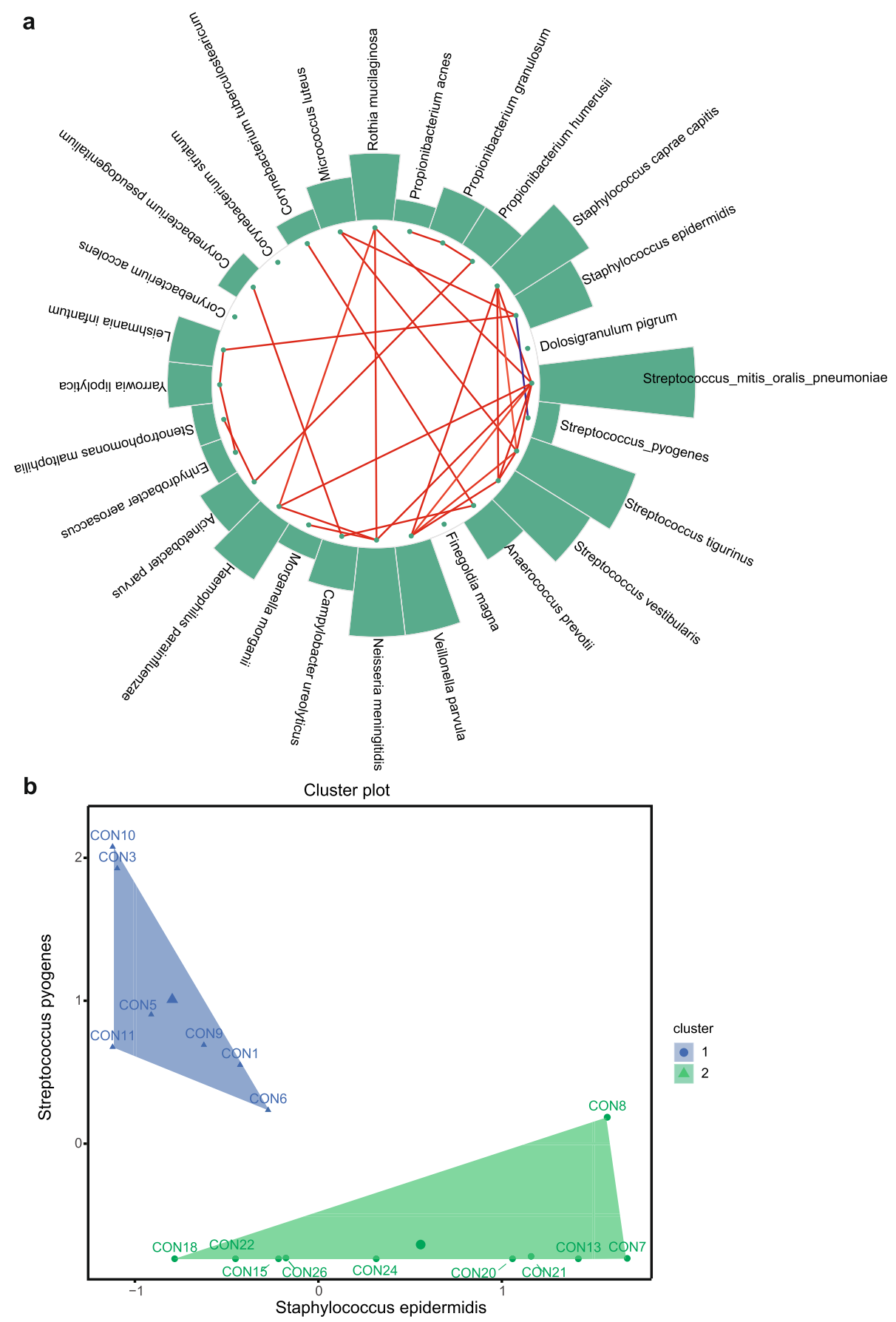

Fig. 4 Microbial cooccurrence in the healthy ocular surface microbiome and K-means clustering for unsupervised grouping. a Interaction network of species with an average relative abundance greater than $0.1 \%$ and appearing in more than two subjects. The colored lines represent correlations between species; red is a positive correlation, and blue is a negative correlation. The heights of the bars are positively correlated with the species' degrees of connectivity. $\mathbf{b}$ K-means clustering $(k=2)$ performed with the relative abundances of Staphylococcus epidermidis and Streptococcus pyogenes

a total of 22 categories (Fig. 5a). Among these, 6 COG functional features were associated with metabolism, namely, amino acid transport and metabolism, nucleotide transport and metabolism, carbohydrate transport and metabolism, coenzyme transport and metabolism, lipid transport and metabolism, and inorganic ion transport and metabolism. Transcription was the most abundant COG annotation, followed by lipid transport and metabolism, signal transduction mechanisms, cell cycle control, cell division, chromosome partitioning and 
a

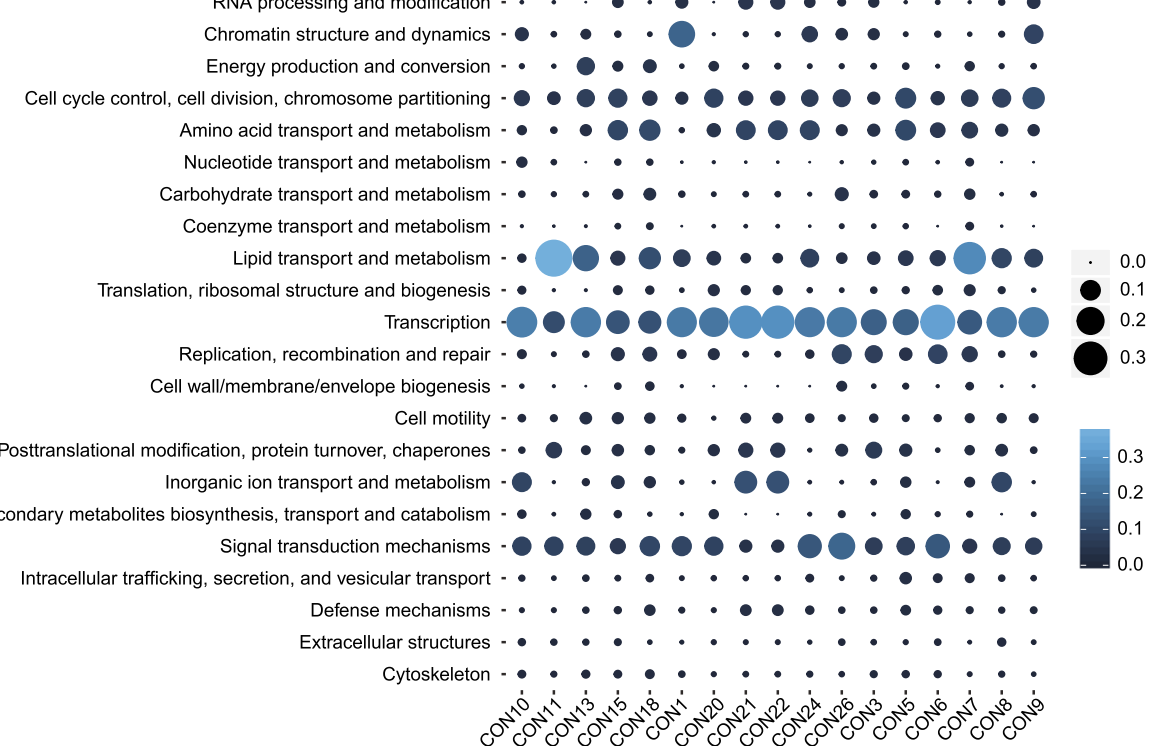

b

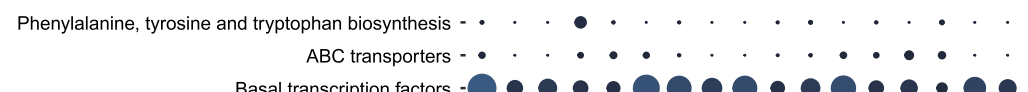

Basal transcription factors $-\bigcirc \bullet \bullet \bullet \bullet \bigcirc \bigcirc \bullet \bigcirc \bullet \bullet \bigcirc \bullet \bullet \bullet \bigcirc \bullet$ Biofilm formation - Escherichia coli -

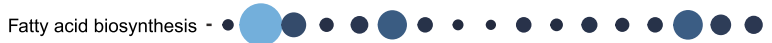

Fig. 5 COG clusters (a) and KEGG (b) pathway heatmap

amino acid transport and metabolism. Figure 5b shows the microbial Kyoto Encyclopedia of Genes and Genomes (KEGG) pathways with a mean relative abundance greater than $0.1 \%$. Similar to the COG annotation results, fatty acid biosynthesis accounted for the largest proportion, followed by basal transcription factors, folate biosynthesis, tyrosine metabolism, and isoquinoline alkaloid biosynthesis. Of note, Escherichia coli biofilm formation and viral carcinogenesis might be potential pathogenic pathways.

\section{Discussion}

The microbial community populated on the human ocular surface may play an important role in both innate and adaptive immune responses. Although these normal commensal florae can colonize the healthy ocular 
surface without causing disease, several studies have revealed that alterations in the healthy ocular surface microbiome are associated with some ocular surface diseases, such as trachoma [12], keratitis [13, 45, 46], conjunctivitis [47], dry eye [48], mesangial gland dysfunction [49, 50], chronic Stevens-Johnson syndrome [51], and blepharitis [52]. Hence, a comprehensive understanding of the composition of the ocular surface microbiome is crucial for the future development of probiotic-based eye treatment drugs. Whereas existing research on the healthy ocular surface microbiome has used mainly $16 \mathrm{~S}$ rRNA gene sequencing, in this study, we performed shotgun metagenomics sequencing on the ocular surface mucosal membrane swabs of 17 healthy volunteers and clearly described the taxonomic composition, the interspecies interactions, strain-level heterogeneity among different individuals, and functional profiles of the healthy ocular surface microbiome.

At the phylum level, the ocular surface microbial community was dominated by Proteobacteria, Firmicutes and Actinomycetes, which is consistent with previous studies $[3,7,10,12,13]$. Only two fungal phyla were found, namely, Ascomycota and Basidiomycota. Compared with a study based on internal transcribed spacer (ITS) sequencing to characterize the ocular surface fungal microbiome [45, 53, 54], we found fewer fungal species; this difference may be due to different sequencing methods. In shotgun metagenomics sequencing, the genomes of eukaryotes are usually very long and composed of many noncoding regions, which leads to poor read utilization [55-57]. For viruses, 5 different strains of Torque teno virus were found on the ocular surface of 5 subjects. Torque teno virus has been identified in culture-negative endophthalmitis [58], but the mechanism of how it causes endophthalmitis remains unclear.

Our results show that age and sex have no effect on the composition of the healthy ocular surface microbiome. There is still controversy about whether sex affects the composition of the ocular surface microbial community, and previous studies have shown mixed results [7, 12, 29, 59]. Future studies should expand the age and sex coverage of subjects and measure their sex hormones, such as estrogen, progesterone, androgens, prolactin, follicle-stimulating hormone, luteinizing hormone, testosterone, progesterone, and estradiol. Exploring the relationship between the level of sex hormones in the body and the diversity of the ocular surface microbial community will better reveal the influence of different sexes on the ocular surface microbiome.

Staphylococcus epidermidis is representative of the normal ocular surface flora and is the species most often isolated from the human eye surface. A previous study on the healthy ocular surface using shotgun metagenomics sequencing reported a high positivity rate for
Staphylococcus epidermidis (73\%) in surveyed subjects [29]. Our data showed a Staphylococcus epidermidis positive rate of $88 \%$. Among these, 10 samples contained enough Staphylococcus epidermidis reads for strain assignment. Phylogenetic tree analysis showed that variation was observed across clades and individual genomes. Earlier studies based on pulsed-field gel electrophoresis also found polyclonality of Staphylococcus epidermidis on the healthy ocular surface [60]. Therefore, it is necessary to perform pangenome sequencing on Staphylococcus epidermidis strains isolated from the healthy ocular surface in the future to evaluate the difference in the function and virulence of different strains.

Interestingly, the healthy ocular surface microbiome of some subjects was dominated by Streptococcus pyogenes, which is a common bacterium isolated from infected eyes. Thus, the healthy ocular surface can achieve homeostasis with causative pathogens. Pathogens that cause eye infections may be introduced from the external environment, and when homeostasis of the eye is disrupted, commensal potential pathogens escape control and become pathogenic $[61,62]$. Whether individuals carrying a high proportion of potential pathogens on the ocular surface are more likely to develop infections requires further research. Phylogenetic analysis showed that there were also individual differences among Streptococcus pyogenes at the strain level, which might be due to host age, living environment, and previous drug use.

A previous study investigated the stability of the composition of the ocular surface microbial community over time [7]. Some genera were present in subjects at all time points (baseline, 1 month, 3 months), which indicated that some taxa have longitudinal stability at the individual level. There is a necessity and is of much interest to study the degree of variability of the strain genomes within an individual over time. Future research should sample the same subject several times in a shorter or longer time interval to reveal the variability degree of the same-strain within-subject changes over time.

Another noteworthy finding is the functional components of the healthy ocular surface microbiome. Both COG and KEGG pathway analyses revealed high abundance of lipid metabolism (biosynthesis, degradation, and transport) pathways. This result may suggest that the healthy ocular surface microbiome plays an important role in lipid metabolism in the eye. The lipid layer can enhance tear distribution, maintain tear stability and prevent evaporation [63]. Recently, lipid formulations have been promoted to regulate dry eye and have achieved good results [64, 65]. Commercially available lipid formulations, including emulsions and liposomes, take the form of eye drops or sprays [66]. Our research provides important ideas for the future development of 
probiotic-based dry eye treatment. In future research, we will perform metagenomic sequencing on the healthy ocular surface microbiome of patients with dry eye to investigate whether the microbial lipid metabolism is reduced.

In addition to lipids, COG annotation results showed that there are also abundant amino acid metabolic pathways on the ocular surface. It has been reported that amino acids could be abundantly produced by Corynebacterium spp. [67]. In our analysis, several Corynebacterium spp. were detected in more than $50 \%$ of the healthy volunteers (Fig. 1a), indicating that Corynebacterium may play a potential role in these amino acid metabolic pathways. The amino acid metabolism pathway in the KEGG annotation results that had the highest proportion was tyrosine metabolism. Amino acids naturally exist in human tears and play a positive role in maintaining ocular surface homeostasis. A series of published clinical data indicate that the use of topical eye drops with amino acids supplemented can be beneficial to the healing of ocular surface diseases [68]. Whether the healthy ocular surface microbiome is involved in the occurrence and role of amino acids in tears is still unknown; a direction worth exploring in future studies.

We also found elevated frequencies of COG annotations related to inorganic ion transport and metabolism. Sphingomyelin are one of the major classes of anionic lipids in human tears [69]. Divalent cations may interact with the phosphate head groups of these phospholipids to help stabilize the lipids in tears [70]. The increase in divalent cations in tear fluid may change the folding of proteins $[71,72]$ and the interaction between proteins, which in turn affect the stability and surface tension of the tear film [73]. Exploring whether the ocular surface microbiome affects the stability of the tear film through the transport and metabolism of inorganic ions has important guiding value for the future development of novel methods that can increase tear film stability and eye comfort.

Probiotics are defined by the World Health Organization (WHO) as "live microorganisms that confer demonstrated health benefits for the host when ingested or topically applied". Some research has been devoted to utilizing probiotics as topical ocular products $[74,75]$. The ocular surface is a wide mucosal surface exposed to the external environment, which contributes largely to the type and number of microorganisms colonizing its surface. As the environment changes, the colonizing organisms may change accordingly. One possibility is that the ocular microbiome is formed by two populations: one stable, tightly embedded population in the ocular surface that is less susceptible to changes and a variable, more superficial population that is sensitive to recent environmental changes, which could be washed away by an ocular rinse.
It is necessary to study whether after a thorough ocular rinse, a more stable microbiome is found in consecutive analyses, which could be relevant for ocular health. Furthermore, this information could suggest what the 'good' microorganisms are to be used in a topical probiotic formulation.

The limitations of this study are as follows. First, the sample size and geographical representation are limited, and thus limits the generalizability of our results to the entire population. Second, the use of anesthetic eye drops before sampling could lead to reduced diversity of ocular surface microbial communities.

\section{Conclusions}

In summary, the healthy ocular surface microbiome was clearly demonstrated by the shotgun metagenomics survey in this study. The present study provides new directions for further studies on the healthy ocular surface microbiome.

\section{Supplementary Information}

The online version contains supplementary material available at https://doi. org/10.1186/s40662-021-00228-4

Additional file 1: Supplemental Figure 1. The number of different classification levels in all samples.

Additional file 2: Supplemental Figure 2. The major taxa with a mean relative abundance greater than $1 \%$ are presented. (a) Major phyla; (b) major genera. "Other" represents groupings of less abundant taxa $(<1 \%)$.

Additional file 3: Supplemental Figure 3. Petal map based on the number of species. The center is the number of species shared by all samples, and the number on the petal shows the number of species specific to each sample.

Additional file 4: Supplemental Figure 4. Alpha and beta diversity of healthy ocular surface microbiota in male and female subjects. Shannon (a) and inverse Simpson indices (b) were used to estimate the level of diversity of the microbiota of the male and female groups. PCoA plots of Bray-Curtis (c) and Jaccard (d) distance matrices between the male and female groups.

Additional file 5: Supplemental Figure 5. Alpha and beta diversity of healthy ocular surface microbiota in young and old subjects. Shannon (a) and inverse Simpson indices (b) were used to estimate the level of diversity of the microbiota of the young and old groups. PCoA plots of Bray-Curtis (c) and Jaccard (d) distance matrices between the male and female groups.

\section{Abbreviations}

NGS: Next-generation sequencing; COG: Clusters of Orthologous Groups: KEGG: Kyoto Encyclopedia of Genes and Genomes; SNPs: Single-nucleotide polymorphisms; PCoA: Principal coordinate analysis; ITS: Internal transcribed spacer; WHO: World Health Organization; PERMANOVA: permutational multivariate analysis of variance; Streptococcus pyogenes CON3: Streptococcus pyogenes residing within CON3; Streptococcus pyogenes CON10: Streptococcus pyogenes residing within CON10

\section{Acknowledgments}

The authors thank Jinyu Wu of the Institute of Genomic Medicine, Wenzhou Medical University for his support and help with data analysis. The authors also thank the Key Discipline of Zhejiang Province in Medical Technology (First Class, Category A). 


\section{Authors' contributions}

Meiqin Zheng and Yongliang Lou contributed to the conception, design, and interpretation of the data. Yutong Kang contributed to data analysis and drafted the article. Shudan Lin, Xueli Ma, Yanlin Che, Yiju Chen, Tian Wan, Die Zhang, Jiao Shao, Jie Xu, and Yi Xu contributed to sample collection and DNA extraction. All authors approved the final version of the manuscript.

\section{Funding}

This work was supported by the National Science and Technology Major Project (Grant No. 2018ZX10201001).

\section{Availability of data and materials}

The datasets used and/or analyzed in this study are available from the corresponding author upon reasonable request.

\section{Ethics approval and consent to participate}

This study was approved by the Ethics Committee of the Eye Hospital of Wenzhou Medical University (number: KYK [2017] 23) and adhered to the tenets of the Declaration of Helsinki. All subjects provided written informed consent at the time of sample collection.

\section{Consent for publication}

Not applicable.

\section{Competing interests}

The authors declare that they have no competing interests.

\section{Author details}

${ }^{1}$ Eye Hospital and School of Ophthalmology and Optometry, Wenzhou Medical University, Wenzhou, Zhejiang, China. ${ }^{2}$ National Clinical Research Center for Ocular Diseases, Wenzhou, Zhejiang, China. ${ }^{3}$ Wenzhou Key Laboratory of Sanitary Microbiology, Key Laboratory of Laboratory Medicine Ministry of Education, School of Laboratory Medicine and Life Sciences, Wenzhou Medical University, Wenzhou, Zhejiang 325035, China.

\section{Received: 15 July 2020 Accepted: 14 January 2021}

\section{Published online: 24 February 2021}

\section{References}

1. Doan T, Akileswaran L, Andersen D, Johnson B, Ko N, Shrestha A, et al. Paucibacterial microbiome and resident DNA virome of the healthy conjunctiva. Invest Ophthalmol Vis Sci. 2016:57(13):5116-26.

2. Galletti JG, Guzmán M, Giordano MN. Mucosal immune tolerance at the ocular surface in health and disease. Immunology. 2017:150(4):397-407.

3. Huang Y, Yang B, Li W. Defining the normal core microbiome of conjunctival microbial communities. Clin Microbiol Infect. 2016;22(7):643.e7643.e12.

4. Teweldemedhin M, Gebreyesus H, Atsbaha AH, Asgedom SW, Saravanan M. Bacterial profile of ocular infections: a systematic review. BMC Ophthalmol. 2017;17:212.

5. Turnbaugh PJ, Ley RE, Hamady M, Fraser-Liggett CM, Knight R, Gordon Jl. The human microbiome project. Nature. 2007;449(7164):804-10

6. Miller D, lovieno A. The role of microbial flora on the ocular surface. Curr Opin Allergy Clin Immunol. 2009;9(5):466-70

7. Ozkan J, Nielsen S, Diez-Vives C, Coroneo M, Thomas T, Willcox M. Tempora stability and composition of the ocular surface microbiome. Sci Rep. 2017; 7(1):9880.

8. Deepthi KG, Jayasudha R, Girish RN, Manikandan P, Ram R, Narendran V, et al. Polybacterial community analysis in human conjunctiva through 165 rRNA gene libraries. Exp Eye Res. 2018;174:1-12.

9. Graham JE, Moore JE, Jiru X, Moore JE, Goodall EA, Dooley JSG, et al. Ocular pathogen or commensal: a PCR-based study of surface bacterial flora in normal and dry eyes. Invest Ophthalmol Vis Sci. 2007:48(12):5616-23.

10. Dong Q, Brulc JM, lovieno A, Bates B, Garoutte A, Miller D, et al. Diversity of bacteria at healthy human conjunctiva. Invest Ophthalmol Vis Sci. 2011; 52(8):5408-13.

11. Schabereiter-Gurtner C, Maca S, Rölleke S, Nigl K, Lukas J, Hirschl A, et al. 165 rDNA-based identification of bacteria from conjunctival swabs by PCR and DGGE fingerprinting. Invest Ophthalmol Vis Sci. 2001;42(6):1164-71.
12. Zhou Y, Holland MJ, Makalo P, Joof H, Roberts CH, Mabey DC, et al. The conjunctival microbiome in health and trachomatous disease: a case control study. Genome Med. 2014;6(11):99.

13. Kang Y, Zhang H, Hu M, Ma Y, Chen P, Zhao Z, et al. Alterations in the ocular surface microbiome in traumatic corneal ulcer patients. Invest Ophthalmol Vis Sci. 2020;61(6):35.

14. Wintermute EH, Silver PA. Dynamics in the mixed microbial concourse. Genes Dev. 2010;24(23):2603-14.

15. Dean SN, Rimmer MA, Turner KB, Phillips DA, Caruana JC, Hervey WJ 4th, et al. Lactobacillus acidophilus membrane vesicles as a vehicle of bacteriocin delivery. Front Microbiol. 2020;11:710.

16. Kumar V, Baweja M, Singh PK, Shukla P. Recent developments in systems biology and metabolic engineering of plant-microbe interactions. Front Plant Sci. 2016;7:1421.

17. Kugadas A, Gadjeva M. Impact of microbiome on ocular health. Ocul Surf. 2016;14(3):342-9.

18. Baim AD, Movahedan A, Faroog AV, Skondra D. The microbiome and ophthalmic disease. Exp Biol Med (Maywood). 2019;244(6):419-29.

19. Cavuoto KM, Banerjee S, Galor A. Relationship between the microbiome and ocular health. Ocul Surf. 2019;17(3):384-92.

20. St Leger AJ, Caspi RR. Visions of eye commensals: the known and the unknown about how the microbiome affects eye disease. Bioessays. 2018; 40(11):e1800046

21. Kugadas A, Christiansen SH, Sankaranarayanan S, Surana NK, Gauguet S, Kunz R, et al. Impact of microbiota on resistance to ocular pseudomonas aeruginosa-induced keratitis. PLoS Pathog. 2016;12(9):e1005855.

22. St. Leger AJ, Desai JV, Drummond RA, Kugadas A, Almaghrabi F, Silver P, et al. An ocular commensal protects against corneal infection by driving an interleukin-17 response from mucosal $\gamma \delta$ T cells. Immunity. 2017;47(1):148-58.e5.

23. Hübner A, Stoneking M. The phylogeographic structure of the strain-level variation of the human oral microbiome. In: EMBL Symposium: The Human Microbiome. Heidelberg: EMBL; 2018.

24. Rosenberg NA, Pritchard JK, Weber JL, Cann HM, Kidd KK, Zhivotovsky $L A$, et al. Genetic structure of human populations. Science. 2002; 298(5602):2381-5

25. Bastos-Rodrigues L, Pimenta JR, Pena SDJ. The genetic structure of human populations studied through short insertion-deletion polymorphisms. Ann Hum Genet. 2006;70(5):658-65.

26. Schloissnig S, Arumugam M, Sunagawa S, Mitreva M, Tap J, Zhu A, et al. Genomic variation landscape of the human gut microbiome. Nature. 2013; 493(7430):45-50

27. Rogier R, Ederveen THA, Wopereis H, Hartog A, Boekhorst J, van Hijum SAFT, et al. Supplementation of diet with non-digestible oligosaccharides alters the intestinal microbiota, but not arthritis development, in IL-1 receptor antagonist deficient mice. PLoS One. 2019;14(7):e0219366.

28. Deng Y, Wen X, Hu X, Zou Y, Zhao C, Chen X, et al. Geographic difference shaped human ocular surface metagenome of young Han Chinese from Beijing, Wenzhou, and Guangzhou cities. Invest Ophthalmol Vis Sci. 2020;61(2):47.

29. Wen X, Miao L, Deng Y, Bible PW, Hu X, Zou Y, et al. The influence of age and sex on ocular surface microbiota in healthy adults. Invest Ophthalmol Vis Sci. 2017:58(14):6030-7.

30. Pakdel F, Gohari MR, Jazayeri AS, Amani A, Pirmarzdashti N, Aghaee H. Validation of farsi translation of the ocular surface disease index. Ophthalmic Vis Res. 2017:12(3):301-4.

31. Wu J, Shen E, Shi D, Sun Z, Cai T. Identification of a novel Cys 146X mutation of SOD1 in familial amyotrophic lateral sclerosis by whole-exome sequencing. Genet Med. 2012;14(9):823-6.

32. Wang T, Liu Q, Li X, Wang X, Li J, Zhu X, et al. RRBS-analyser: a comprehensive web server for reduced representation bisulfite sequencing data analysis. Hum Mutat. 2013;34(12):1606-10.

33. Liu Q, Chen C, Shen E, Zhao F, Sun Z, Wu J. Detection, annotation and visualization of alternative splicing from RNA-Seq data with SplicingViewer. Genomics. 2012:99(3):178-82.

34. Langmead B, Salzberg SL. Fast gapped-read alignment with bowtie 2. Nat Methods. 2012:9(4):357-9.

35. Li H, Handsaker B, Wysoker A, Fennell T, Ruan J, Homer N, et al. The sequence alignment/map format and SAMtools. Bioinformatics. 2009;25(16):2078-9.

36. Truong DT, Franzosa EA, Tickle TL, Scholz M, Weingart G, Pasolli E, et al. MetaPhIAn2 for enhanced metagenomic taxonomic profiling. Nat Methods. 2015;12(10):902-3. 
37. Truong DT, Tett A, Pasolli E, Huttenhower C, Segata N. Microbial strain-level population structure and genetic diversity from metagenomes. Genome Res. 2017;27(4):626-38

38. Li DH, Liu CM, Luo R, Sadakane K, Lam TW. MEGAHIT: an ultra-fast singlenode solution for large and complex metagenomics assembly via succinct de Bruijn graph. Bioinformatics. 2015;31(10):1674-6.

39. Seemann T. Prokka: rapid prokaryotic genome annotation. Bioinformatics. 2014;30(14):2068-9.

40. Patro R, Duggal G, Love MI, Irizarry RA, Kingsford C. Salmon provides fast and bias-aware quantification of transcript expression. Nat Methods. 2017; 14(4):417-9.

41. Fu L, Niu B, Zhu Z, Wu S, Li W. CD-HIT: accelerated for clustering the nextgeneration sequencing data. Bioinformatics. 2012;28(23):3150-2.

42. Huerta-Cepas J, Forslund K, Coelho LP, Szklarczyk D, Jensen LJ, von Mering $C$, et al. Fast genome-wide functional annotation through orthology assignment by eggNOG-mapper. Mol Biol Evol. 2017;34(8):2115-22.

43. Kilic S, White ER, Sagitova DM, Cornish JP, Erill I. CollecTF: a database of experimentally validated transcription factor-binding sites in Bacteria. Nucleic Acids Res. 2014;42:D156-60

44. Wu J, Wang S, Bai J, Shi L, Li D, Xu Z, et al. ArchaeaTF: an integrated database of putative transcription factors in Archaea. Genomics. 2008; 91(1):102-7

45. Prashanthi GS, Jayasudha R, Chakravarthy SK, Padakandla SR, SaiAbhilash CR, Sharma $S$, et al. Alterations in the ocular surface fungal microbiome in fungal keratitis patients. Microorganisms. 2019;7(9):309.

46. Ge C, Wei C, Yang BX, Cheng J, Huang YS. Conjunctival microbiome changes associated with fungal keratitis: metagenomic analysis. Int Ophthalmol. 2019;12(2):194-200.

47. Yau JWK, Hou JP, Tsui SKW, Leung TF, Cheng NS, Yam JC, et al. Characterization of ocular and nasopharyngeal microbiome in allergic rhinoconjunctivitis. Pediatr Allergy Immunol. 2019;30(6):624-31.

48. Hori Y, Maeda N, Sakamoto M, Koh S, Inoue T, Tano Y. Bacteriologic profile of the conjunctiva in the patients with dry eye. Am J Ophthalmol. 2008; 146(5):729-34

49. Jiang XD, Deng AH, Yang JR, Bai H, Yang Z, Wu J, et al. Pathogens in the Meibomian gland and conjunctival sac: microbiome of normal subjects and patients with Meibomian gland dysfunction. Infect Drug Resist. 2018;11: 1729-40.

50. Zhao F, Zhang D, Ge C, Zhang L, Reinach PS, Tian X, et al. Metagenomic profiling of ocular surface microbiome changes in meibomian gland dysfunction. Invest Ophthalmol Vis Sci. 2020;61(8):22.

51. Kittipibul T, Puangsricharern V, Chatsuwan T. Comparison of the ocular microbiome between chronic Stevens-Johnson syndrome patients and healthy subjects. Sci Rep. 2020;10(1):4353.

52. Lee SH, Oh DH, Jung JY, Kim JC, Jeon CO. Comparative ocular microbial communities in humans with and without blepharitis. Invest Ophthalmol Vis Sci. 2012:53(9):5585-93.

53. Shivaji S, Jayasudha R, Sai Prashanthi G, Kalyana Chakravarthy S, Sharma S. The human ocular surface fungal microbiome. Invest Ophthalmol Vis Sci. 2019;60(1):451-9.

54. Wang Y, Chen $\mathrm{H}$, Xia T, Huang Y. Characterization of fungal microbiota on normal ocular surface of humans. Clin Microbiol Infect. 2020;26(1):123.e9-e13.

55. Hugenholtz P, Tyson GW. Microbiology: metagenomics. Nature. 2008; 455(7212):481-3.

56. Cowan D, Meyer Q, Stafford W, Muyanga S, Cameron R, Wittwer P. Metagenomic gene discovery: past, present and future. Trends Biotechnol. 2005;23(6):321-9.

57. Gilbert JA, Dupont CL. Microbial metagenomics: beyond the genome. Annu Rev Mar Sci. 2011;3:347-71.

58. Lee AY, Akileswaran L, Tibbetts MD, Garg SJ, Van Gelder RN. Identification of torque teno virus in culture-negative endophthalmitis by representational deep DNA sequencing. Ophthalmology. 2015;122(3):524-30.

59. Shin H, Price K, Albert L, Dodick J, Park L, Dominguez-Bello MG. Changes in the eye microbiota associated with contact lens wearing. mBio. 2016;7(2):e00198.

60. Ueta M, lida T, Sakamoto M, Sotozono C, Takahashi J, Kojima K, et al. Polyclonality of Staphylococcus epidermidis residing on the healthy ocular surface. J Med Microbiol. 2007;56(Pt 1):77-82.

61. Rishi E, Rishi P, Koundanya W, Sahu C, Roy R, Bhende PS. Post-traumatic endophthalmitis in 143 eyes of children and adolescents from India. Eye (Lond). 2016;30(4):615-20.
62. Creuzot-Garcher C, Benzenine E, Mariet AS, de Lazzer A, Chiquet C, Bron AM, et al. Incidence of acute postoperative endophthalmitis after cataract surgery a nationwide study in France from 2005 to 2014. Ophthalmology. 2016:123(7):1414-20.

63. Foulks GN. The correlation between the tear film lipid layer and dry eye disease. Surv Ophthalmol. 2007:52(4):369-74.

64. Khanal S, Tomlinson A, Pearce El, Simmons PA. Effect of an oil-in-water emulsion on the tear physiology of patients with mild to moderate dry eye. Cornea. 2007:26(2):175-81.

65. Di Pascuale MA, Goto E, Tseng SCG. Sequential changes of lipid tear film after the instillation of a single drop of a new emulsion eye drop in dry eye patients. Ophthalmology. 2004;111(4):783-91.

66. Lim A, Wenk MR, Tong L. Lipid-based therapy for ocular surface inflammation and disease. Trends Mol Med. 2015;21(12):736-48.

67. Liu G, Wu J, Yang H, Bao O. Codon usage patterns in Corynebacterium glutamicum: mutational bias, natural selection and amino acid conservation. Comp Funct Genomics. 2010;2010:343569.

68. Rusciano D, Roszkowska AM, Gagliano C, Pezzino S. Free amino acids: an innovative treatment for ocular surface disease. Eur J Pharmacol. 2016:787:9-19.

69. Saville JT, Zhao Z, Willcox MD, Blanksby SJ, Mitchell TW. Detection and quantification of tear phospholipids and cholesterol in contact lens deposits: the effect of contact lens material and lens care solution. Invest Ophthalmol Vis Sci. 2010:51(6):2843-51.

70. Lösche M, Möhwald H. Electrostatic interactions in phospholipid membranes: II. Influence of divalent ions on monolayer structure. J Colloid Interface Sci. 1989;131:56-67.

71. Yusifov TN, Abduragimov AR, Gasymov OK, Glasgow BJ. Endonuclease activity in lipocalins. Biochem J. 2000;347(Pt 3):815-9.

72. Tiwari S, Askari JA, Humphries MJ, Bulleid NJ. Divalent cations regulate the folding and activation status of integrins during their intracellular trafficking. J Cell Sci. 2011;124(Pt 10):1672-80.

73. Wei XE, Markoulli M, Millar TJ, Willcox MD, Zhao Z. Divalent cations in tears, and their influence on tear film stability in humans and rabbits. Invest Ophthalmol Vis Sci. 2012;53(7):3280-5.

74. Mangiafico S, Aleo D, Saita MG, Cro MG, Mangiafico S. A stable aqueous formulation of probiotics for topical ocular therapy. Invest Ophthalmol Vis Sci. 2012;53(14):2757

75. Iovieno A, Lambiase A, Sacchetti M, Stampachiacchiere B, Micera A, Bonini S. Preliminary evidence of the efficacy of probiotic eye-drop treatment in patients with vernal keratoconjunctivitis. Graefes Arch Clin Exp Ophthalmol. 2008:246(3):435-41.
Ready to submit your research? Choose BMC and benefit from:

- fast, convenient online submission

- thorough peer review by experienced researchers in your field

- rapid publication on acceptance

- support for research data, including large and complex data types

- gold Open Access which fosters wider collaboration and increased citations

- maximum visibility for your research: over $100 \mathrm{M}$ website views per year

At $\mathrm{BMC}$, research is always in progress.

Learn more biomedcentral.com/submission 